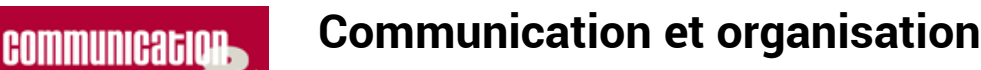

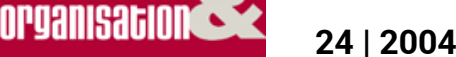

Coexister dans les mondes organisationnels

\title{
La communication interculturelle : ses fondements, les obstacles à son développement
}

Bo Shan

\section{OpenEdition}

12 Journals

Édition électronique

URL : http://journals.openedition.org/communicationorganisation/2928

DOI : 10.4000/communicationorganisation.2928

ISSN : $1775-3546$

Éditeur

Presses universitaires de Bordeaux

Édition imprimée

Date de publication : 1 mai 2004

ISSN : 1168-5549

Référence électronique

Bo Shan, "La communication interculturelle : ses fondements, les obstacles à son développement », Communication et organisation [En ligne], 24 | 2004, mis en ligne le 27 mars 2012, consulté le 10 décembre 2020. URL : http://journals.openedition.org/communicationorganisation/2928 ; DOI : https://doi.org/10.4000/communicationorganisation.2928

Ce document a été généré automatiquement le 10 décembre 2020.

(c) Presses universitaires de Bordeaux 


\title{
La communication interculturelle : ses fondements, les obstacles à son développement
}

\author{
Bo Shan
}

1 La communication interculturelle est un phénomène historiquement culturel: accompagnant le développement de l'être humain, elle constitue également un mode de vie de celui-ci. Selon ce que disent les anthropologues culturels, la ressemblance culturelle de chaque nation pourrait s'expliquer en grande partie, même si pas en totalité, par la communication ou "l'emprunt » qui s'est passée dans l'histoire par le contact. Sinon la culture descend d'une origine commune. F. Graebner anthropologue culturel allemand, pense que la distance de deux zones culturelles ne peut pas empêcher la communication interculturelle que ces zones soient voisines ou éloignées, car il existe une "vague culturelle " mystérieuse. Il nous paraît difficile de comprendre la «vague culturelle " comme un phénomène historique, par laquelle nous sommes vraiment frappés dans notre vie réelle: l'habitude et la coutume culturels séparent les uns des autres, mais d'innombrables « autrui » et « étrangers » culturels entrent en contact avec nous. Nous nous connaissons et nous réglons afin de chercher la voie d'existence et de développement en observant et en étant observés, en comprenant et en étant compris, en acceptant et en étant acceptés, en modifiant et en étant modifiés. Le groupe culturel auquel nous appartenons est fondé sur l'âge, le sexe, la famille et la race, et aussi sur des critères d'alliance professionnelle ou politique ou de goût, sur la religion, la nationalité ou l'état économique et social. Parmi ces groupes contrastés, l'interaction culturelle serait soit positive, ou intéressante, soit négative, ou sans intérêt. Quelle est la base de la communication interculturelle? Par quel moyen pourrions-nous surmonter l'obstacle afin de réaliser celle qui est positive ? À quoi est confronte l'individu moderne.

2 Lu général, la communication interculturelle est constituée par l'interaction de l'homme dans le contexte des cultures différentes. Si avec le sociologue allemand Georg Simmel on introduit la notion "d'étranger" la communication interculturelle concerne, sur le niveau fondamental, la relation de l'individu avec l'étranger. L'observation que Simmel a 
effectuée à l'égard du rôle de l'étranger fait partie de sa réflexion générale au sujet de la relation entre des individus. À ses propres veux, la communication est une forme fondamentale de la vie sociale. La société est constituée par la communication entre des individus. Une certaine forme de la communication deviendra relativement stable après un certain temps et constituera donc la structure culturelle et sociale, qui influencera l'interaction des individus. Quand un individu est intégré dans un groupe, il a l'obligation d'abandonner une certaine individualité pour demeurer se mettre en accord avec la norme du groupe, afin que le partage des valeurs soit réalisé et qu'un certain système de valeur culturelle soit formé. Ainsi les membres du groupe se jugent-ils réciproquement à travers la "perspective » culturelle de groupe au lieu de le faire avec un critère objectif : c'est à ce moment-là qu'intervient « l'étranger » qui diffère du système culturel et qui n'est pas totalement accepté par des membres de groupe. Cela signifie que le système culturel où nous nous trouvons nous distribue des rôles dans le groupe et celui de "l'étranger " à un autre. L'étranger nous apporte un avantage original avec son recul tout en observant de manière différente le système culturel et social où nous nous trouvons. Il est moins contraint à maintenir une liberté d'y accéder et de s'en dégager tout en traversant la frontière et en vivant aux bornes de groupes différents. Il lai est facile de regarder le problème en cours de communication avec l'angle de vision interculturelle quand il identifie une certaine forme de symboles. Il ne pourrait pas les lier immédiatement à un sens particulier. En même temps, comme les conduites de "l'étranger" s'avèrent incertaines et imprévisibles, on a toujours des doutes sur « l'étranger » les conséquences extrêmes étant la xénophobie et le conflit.

Dans la vie réelle, nous nous intégrons de façon consciente ou inconsciente dans le groupe culturel diversifié. Nous rencontrons souvent l'étranger ou nous sommes considérés comme l'étranger. La plupart du temps, l'homme réalise le partage des modes de vie des mœurs, des goûts, des sensations, des valeurs et des croyances dans et par la communication. Cela permet de former un groupe culturel. Cependant, bien que nous ayons l'appartenance culturelle, nous ne pourrons pas réaliser de manière évidente la cognition du groupe culturel auquel nous appartenons. Par exemple, il est difficile pour un Chinois d'expliquer clairement ce qu'est la culture chinoise, et il en va de même pour un Américain. Nous ne sommes pas en mesure de confirmer nos propres caractéristiques dans un cadre de repérage culturel jusqu'à ce que nous rencontrions et contactions un individu provenant d'une autre culture. Dans ce sens, la communication interculturelle provient tout d'abord de la différence culturelle et du sentiment d'inconnu parmi les hommes, cela veut dire qu'elle est née entre des individus qui possèdent mutuellement une distance culturelle. En cette période, la communication culturelle manifeste davantage un besoin de sa propre réalisation de l'être humain, un besoin de la curiosité de création et d'originalité, un besoin de l'étendue de relations spirituelles à travers la réalisation de la connaissance d'autrui fous ces besoins constituent en fin de compte un motif psychologique interne et également une partie importante des caractéristiques interculturelles de l'homme. Il est normal que la communication entraîne aussi le conflit, la concurrence, la tension et la nervosité jusqu'à l'instabilité du système culturel et social. Cette caractéristique constitue elle-même une partie inséparable de la tendance des relations humaines.

4 L'histoire de la communication humaine montre que le besoin de relations spirituelles a été produit depuis que l'homme a créé une vie matérielle. Par exemple, les peintures que l'on trouve sur les murs des grottes sont considérées comme des preuves des relations 
spirituelles de l'être humain préhistorique. Selon le point de vue marxiste, l'histoire de l'homme a débuté avec la création de sa propre $v$ ie matérielle et a évolué dans la relation matérielle entre les hommes. Cependant comme le besoin de relation spirituelle a vu le jour dès la création de la vie matérielle, l'homme a créé sans cesse les relations spirituelles avec les matérielles. Les relations de l'homme se sont alors généralisées avec le développement général de la productivité, de telle sorte que l'individu qui était essentiellement local a été remplacé par l'individu historiquement mondialisé et généralisé. C'est notamment après la révolution industrielle que le développement général de la productivité et la division de travail ont entraîné les relations généralisées de toutes les nations et ont poussé l'homme à entrer dans une telle scène historique : la satisfaction des besoins de chacun dépend du monde entier. La compréhension et les relations mutuelles au niveau inter-local et interculturel aident l'ouverture de soi-même et de la société afin de mieux satisfaire les besoins de l'homme. De nos jours, la globalisation économique a détruit rapidement les caractéristiques qui s'avéraient locales et bien fermées. Elle permet non seulement aux commerçants voyageant dans le monde entier de jouer le rôle de «l'étranger » mais aussi à toutes les personnes imprégnées de la civilisation moderne de manifester davantage de caractéristiques interculturelles. Il est évident que la communication interculturelle est plantée dans le besoin général de relation matérielle et à la fois spirituelle de l'homme dans le domaine historique.

Cependant, la base de la communication interculturelle n'est pus disposée par l'homme. En lin de compte, la relation matérielle suivie du monopole et de la pénétration des capitaux et la relation spirituelle comprenant en cachette la discrimination, le préjugé et la domination d'esprit pourraient déformer la communication interculturelle. La réalité que nous pouvons toucher est que la globalisation des capitaux a entraîné le lax du pouvoir politique, économique et culturel en sens unique et le contrôle unilatéral, qui constituent une menace pour la diversité culturelle. Evidemment, la communication interculturelle doit reposer sur un régime complet de communication et d'éthique interculturelle afin que la communication entre des personnes et les groupes culturels manifeste non seulement une diversité culturelle, soit la nature de la diversité de l'homme, mais encore contrôle la création d'hégémonie culturelle et l'infiltration du bénéfice de groupe. En ce qui concerne le soi-disant régime complet de communication, il s'agit de la formation au partage de valeurs ainsi que l'opposition, la négociation et l'acceptation en cours de partage. Nous parlons ici du problème constructif entre l'hétérogénéité cl l'homogénéité. En général, la communication a lieu plus facilement parmi des individus homogènes, et celle qui est homogène dispose davantage d'influences que celle qui est hétérogène. La communication efficace entre des individus conduit plus à la création homogène en matière de connaissance, d'attitude et de comportement. C'est en détruisant la différence entre les individus et en faisant devenir absolument unilatérale et partiale la communication homogène de l'être humain qu'elle force finalement l'homme à accepter la domination homogène. À vrai dire, une communication significative se déroule entre les individus qui se situent dans la différenciation culturelle et sociale. Le processus du partage de valeurs présente le sens bilatéral de l'homogénéité et l'hétérogénéité. Derrière celui de la communication significative, il existe un problème d'éthique interculturelle, c'est-à-dire que la communication interculturelle possédant la valeur humaine devrait se traduire par un processus d'interaction entre les états, les nations et les cultures. C'est grâce â cette interaction que tout état, toute nation et toute culture a rompu sa propriété unilatérale, sa limite et son opposition tout en maintenant ses caractéristiques du développement de soi-même. Ainsi a été créée la structure 
multipolaire et diversifiée telle que la dépendance, le respect et la communication réciproques. L'éthique interculturelle signifie donc le respect de la personnalité culturelle de l'être humain et des caractéristiques interculturelles, la stimulation de l'ouverture culturelle, du renouvellement et de la diversité illimités des modes d'expression culturelle, la résistance à l'homogénéité culturelle, l'encouragement du partage des informations et de la valeur entre des régions culturelles, la résistance au pouvoir dominant impérialiste, la protection de la valeur de la multiculture : tout cela a pour objectif d'exprimer que la communication interculturelle est destinée à l'être humain.

Nous fondant sur cette connaissance, nous pouvons mettre en évidence que l'obstacle de la communication interculturelle ne touche pas la distance entre des individus et la culture sociale, ni le sentiment étranger d'un individu envers «l'étranger» ni le conflit entre les cultures. Il s'agit plutôt du régime de la communication étroite provoquée par l'homogénéité ainsi que du mode de vie homogène et de pensée formé en cours de la compréhension et de de la domination d'une autre culture. Par exemple, le centralisme national, le préjugé, la discrimination et le stéréotype.

En ce qui concerne le système de la communication dite étroite, il s'agit d'une part de la communication unilatérale et partiale de l'information réalisée sur la base de la domination de pensée et du mode de fonctionnement du pouvoir centraliste, et d'autre part du flux et du contrôle unilatéral du pouvoir politique, économique et culturel, afin que la personnalité, la limite et la diversité culturelle disparaissent avec la naissance d'une société internationale virtuelle, dominée par des capitaux et « un sentiment à la mode » (un standard culturel). La première provoque facilement le dégoût et l'opposition de l'homme moderne contre le régime de la communication féodalisée. L'autre fait tomber l'homme dans la standarisation et la conformité de la création culturelle avec une logique de capitaux et une fièvre stupéfiante de consommation. En tous cas elle détruira la base de la communication interculturelle. Le soi-disant centralisme se traduit par une foi. c'est-à-dire qu'une personne pense que le groupe auquel elle appartient elle-même en général l'équivalent de l'état - est supérieur à celui provenant d'autres cultures. Il représente le complexe de supériorité nationale, soit le jugement d'autres valeurs culturelles à partir de celles de sa propre culture nationale. Elle pense que la valeur d'autres cultures nationales est inférieure à celle de sa propre nation. Parmi les hommes, il n'y a personne qui possède dès sa naissance un complexe de supériorité national et une foi dans le centralisme national. Il est évident que l'homme les a acquis dans le groupe culturel. Si la culture signifie le mode d'apprentissage et de partage de la vision du monde, elle pourra être décrite comme « la perspective » à travers laquelle nous pourrons juger le monde et effectuer le choix, l'évaluation et l'organisation d'informations dans un environnement extérieur. La foi en sa propre culture se forme de manière naturelle. Il faut dire que la confiance en sa propre culture n'est pas mauvaise et qu'elle favorise au cours de la communication la transmission de la valeur estimée importante. Cependant, le centralisme national qui semble extrême pense qu'un individu ne peut pas croire à d'autres bonnes valeurs culturelles. Quand le centralisme national empêche des individus de connaître les points de vue d'autrui à travers une autre « perspective » culturelle, il est devenu un obstacle. Sa pensée typique est que la plupart des cultures sont inférieures à la sienne. Elle doit donc servir d'exemple aux autres et il n'est pas nécessaire de respecter les valeurs et les mœurs des autres cultures. Selon les enquêtes de toutes sortes, nous nous habituons à nous valoriser en valorisant le groupe culturel auquel nous appartenons. Par conséquent, nous avons davantage tendance à accepter la valeur 
culturelle du groupe, à observer "l'autre " culturel en utilisant la «perspective " culturelle attribuée par le groupe, à ne pas vouloir examiner notre propre valeur culturelle et à perdre la capacité à nous examiner à l'intérieur de notre propre culture. Malheureusement, la supériorité nationale est presque toujours contenue dans la communication interculturelle dont elle réduit l'efficacité, car cela entraîne souvent l'évincement réciproque entre l'individu et le groupe interculturel de telle sorte que l'échange de la communication de valeur ne peut pas être réalisable.

En même temps, le stéréotype, le préjugé et la discrimination sont accompagnés du centralisme national. Ils constituent tous ensemble l'obstacle pour la communication interculturelle, le stéréotype est une connaissance schématisée sur une autre culture formée par la synthétisation trop simplifiée quand nous recevons dans la vie quoditienne les informations d'une autre culture. Cette connaissance conceptuelle l'orme une impression stéréotypée envers d'autres cultures. La synthèse d'une autre culture aurait été un mode d'interaction dans la communication interculturelle. La conceptualisation signifie également que l'homme cherche à connaître la certitude d'une autre culture. Une fois l'impression stéréotypée formée, l'usure de l'information apparait dans l'interaction entre des groupes de cultures différentes et influence ainsi la connaissance exacte et totale en provoquant le préjugé. Le préjugé est un jugement irrationnel sur une autre culture sans avoir obtenu l'information exacte et complète, et l'attitude niant l'autre culture se forme ainsi. Ce qui est affreux dans le préjugé, ce n'est pas le préjugé même, mais le système psychologique de la culture sociale caché par le préjugé. Un individu qui a préjugé contre celui qui provient d'un groupe d'une autre culture a quelquefois pour but de se dissimuler. Par exemple, un professeur incompétent a facilement préjugé contre un groupe d'élèves afin de dissimuler son incompétence. Il a aussi pour objectif de renforcer une certaine foi ou valeur, car un être humain a préjugé contre une autre culture. Voyons un autre exemple, un individu appartenant à un certain groupe religieux aura préjugé contre une autre religion pour promouvoir sa propre croyance religieuse. Si le préjugé est une attitude, la discrimination constitue une action. Quand l'attitude niant une autre culture se transforme en une action, cette action est appelée "la discrimination » soit une manière de traiter un individu injustement en termes de nation, de sexe, d'âge et de profession. La discrimination la plus affreuse est celle d'un groupe envers un autre (un blanc envers un noir, une nation envers une autre), car la discrimination deviendrait légale dès qu'elle constitue une inconscience collective.

En conséquence le centralisme national, le stéréotype, le préjugé et la discrimination constituent un outil d'analyse sur l'obstacle à la communication interculturelle. Cet outil peut nous aider à examiner la dérive de la structure culturelle de la société et l'irrationalité de l'échange culturel et à connaître l'essence de la communication interculturelle pour qu'elle soit plus complète et plus libre. Dans un sens, l'examen positif cache une réponse à la question suivante : comment surmonter l'obstacle et réaliser la communication interculturelle dynamique. 


\section{BIBLIOGRAPHIE}

XIANG. Li Hui. Amtropologie culturelle. Edition Shang Wu. 1991.

ROGERS, Everett M., STEINFATE Thomas M. Intercultural Communication. Waveland Press. 1999.

MARTIN. Judith N. NAKAYAMA Thomas K. Experiencing Intercultural Communication An Introduction. Mayfield Publishing Company.2001 